\title{
Analgesic effect of intrathecal fentanyl as an adjuvant to spinal anaesthesia in comparison with spinal anaesthesia with bupivacaine only for mothers delivered by emergency cesarean section
}

\begin{abstract}
Background: Pain during cesarean section is a common phenomenon which results release of stress hormone during surgery. In contrast, effective analgesia may permit improved mother-child bonding, early ambulation, discharge, greater patient satisfaction and early breastfeeding. Now a days the use of intrathecal fentanyl as multimodal analgesia became a popular technique for cesarean section, because of synergistic effect with local anaesthetic and intensify sensory block without increasing sympathetic block. This study aimed to assess postoperative analgesic effect of intrathecal fentanyl as an adjuvant to spinal anaesthesia in comparison with spinal anaesthesia with bupivacaine only among laboring mothers who undergone emergency cesarean delivery.
\end{abstract}

Methods: A hospital based observational cohort study was conducted by recruited 100 patients scheduled for emergency cesarean section and grouped in to BS group, $12.5 \mathrm{mg}$ bupivacaine only $(\mathrm{n}=50)$ and $\mathrm{BF}$ group, $25 \mathrm{mcg}$ fentanyl with $10 \mathrm{mg}$ bupivacaine $(\mathrm{n}=50)$. Patient's vital signs were taken intraoperativley. Postoperatively duration \& consumption of analgesia, first analgesia request as well as severity of pain using $100 \mathrm{~mm}$ VAS score were assessed over $12 \mathrm{hrs}$. Normally distributed data analyzed using student $\mathrm{t}$ test and none parametric data was analyzed using Mann-Whitney U test with $95 \% \mathrm{CI}$ and p- value less than 0.05 is considered as statistically significant.

Results: Bupivacainefentanyl group $(\mathrm{BF}, \mathrm{n}=50)$ compared with bupivacaine only group (BS, $\mathrm{n}=50)$, total duration of analgesia was longer in BF group (275.10 \pm 42.43$)$ than BS group $(156.10 \pm 34.45)$ in minutes, $P=0.001$, analgesia request time in $\mathrm{BF}(301.00 \pm 46.55)$ vs. (200.20 \pm 44.19$)$ in minute in BS group, $P=0.001$ also longer. Not only this but also total amount of tramadol consumption 50(50)mg in BF vs. $100(50) \mathrm{mg}$ in BS, $P=0.001$. VAS score also reduced at $2 \mathrm{hrs}, 3 \mathrm{hrs}, 4 \mathrm{hr}, 5 \mathrm{hrs}$ and $6 \mathrm{hrs}$ in BF group. None of group BS patients developed pruritus but 7(14\%) in group BF developed mild pruritus, which did not need treatment. Shivering occurred $15(30 \%)$ patients in BF vs. 29(58\%) in BS group with p- value of 0.005 .

Conclusion: Addition of intrathecal fentanyl as an adjuvant to bupivacaine for emergency cesarean section increased duration of analgesia, reduced postoperative analgesia consumption and request time without any maternal and fetal effect. We recommend the use of intrathecal fentanyl for emergency cesarean section in our set up.

Keywords: spinal anaesthesia, cesarean delivery, intrathecal fentanyl, postoperative analgesia
Volume 7 Issue 5 - 2017

\section{Kassaw Abegaz Yesuf, Endale Gebreegziabher Gebremedhn, Tadesse Belayneh Melkie \\ Department of Anaesthesia, School of Medicine, Gondar College of Medicine and Health Sciences, The University of Gondar, Ethiopia}

Correspondence: Endale Gebreegziabher, Department of Anaesthesia, School of Medicine, Gondar College of Medicine and Health Sciences, the University of Gondar, Gondar-Ethiopia, P.O.Box:196, Tel +251910880 I 17, Email endalege21@gmail.com

Received: February 02, 2017 | Published: April 13, 2017

\section{Introduction}

Caesarean delivery (CD) can be the best way to insure the safety of the mother and the baby especially, if the baby is in distress in the later stage of pregnancy. ${ }^{1}$ The choice of anaesthesia for caesarean section depends on the reason for the operation, degree of urgency, the desires of the patient and the judgment of anesthesiologist. ${ }^{2}$ General anaesthesia for the CD is associated with relatively greater maternal risk than regional anaesthesia. ${ }^{3}$ Spinal Anaesthesia (SA) has therefore become more widely practiced anaesthetic technique in CD. It is simple to institute, rapid in its effect and produces excellent operating conditions. It also avoids fetal as well as maternal risks of GA, requires minimum postoperative anaesthesia care and provides adequate postoperative analgesia. ${ }^{4}$

Bupivacaine is the most commonly used drug worldwide for spinal anaesthesia in CD. Its effect lasts longer than other local anaesthetists with minimal side effects and it is also affordable. Spinal anaesthesia with local anaesthetic agents, especially bupivacaine, has side effects such as hypotension, respiratory depression, vomiting and shivering in a dose dependent fashion ${ }^{5}$ (Jaishri B, 2005 \#34). Hypotension is one of the commonest side effects and can affect both the mother and the fetus or the neonate. Its side effects are dose dependent, therefore different approaches have been attempted in order to avoid spinalinduced complication including the use of small dose of bupivacaine $e^{6,7}$ or by lowering the dose of local anaesthetic and mixing it with additives like neuraxial opioids. ${ }^{8}$

With isobaric bupivacaine the sensory blockade reaching may be insufficient and additional intraoperative analgesia is required. ${ }^{9,10}$ Higher doses of bupivacaine, increasing the level of blockade are likely to enhance hypotension and induce breathing difficulties but reducing the dose of bupivacaine does not prevent hypotension ${ }^{11}$. So coadministration of small doses of intrathecal opioids with bupivacaine for spinal anaesthesia is advisable and advantageous in order to 
decrease the intensity as well as severity of spinalcomplications associated with spinal anaesthesia., ${ }^{9,12}$

Cesarean delivery requires traction of peritoneum and handling of intraperitoneal organs, resulting in intraoperative visceral pain which is a poorly localized type of pain from deep structures in the body. With higher doses of hyperbaric bupivacaine the incidence of intraoperative visceral pain is reduced, but increasing the dose of bupivacaine increases the risk of high block. ${ }^{13}$ Postoperative pain after CD is an unpleasant outcome for women and may result in delayed ambulation, prolonged time for discharge from hospital, poor bonding with the newborn, low satisfaction scores and delay breastfeeding. In addition to this inadequate analgesia leads to elevated plasma catecholamine concentrations, resulting in adverse effect on all organ systems. ${ }^{14}$ In contrast, effective analgesia may permit improved mother - child bonding, early ambulation and discharge, greater patient satisfaction and early breastfeeding..$^{15}$ Neuraxial administration of opioids along with local anesthetics improves the quality of intraoperative analgesia and provides postoperative pain relief for a longer duration than local anaesthetics alone. ${ }^{16,17}$ Fentanyl is a synthetic lipophilic opioid with a rapid onset of action and, unlike morphine, has fewer tendencies to migrate rostrally to the fourth ventricle in sufficient concentration to cause delayed respiratory depression. Intrathecal fentanyl has faster onset of action, it improves quality of intraoperative analgesia, reduces intrathecal doses of local anaesthetic drugs and is associated with less side effects and good postoperative analgesia. ${ }^{18}$

A randomized control trial study by Dhumal PR et al to determine effectiveness of sensory and motor blockade after spinal anaesthesia with plain bupivacaine $(7.5 \mathrm{mg}$ of $0.5 \%)$ and bupivacaine $(5 \mathrm{mg}$ of $0.5 \%$ Bupivacaine) with fentanyl $(25 \mathrm{mcg})$ for elective cesarean section shows the mean time required to reach peak sensory level and duration of motor recovery time was earlier in Group BF than Group B $(P=<0.001)$ but, mean time of two segment regression of sensory analgesia and complete sensory recovery was significantly early in Group B $(P=<0.05)$. This shows the duration of effective analgesia was significantly more in Group BF $(225.3 \pm 29.2)$ compared with control group $(110.33 \pm 8.90)$.

In another similar study in Texas showsthe addition of fentanyl with bupivacaine reduced postoperative analgesia consumption by $28 \%$ for 143 minutes. ${ }^{19}$ In addition to this mean maximal pulse rate $(103.60 \pm 6.92$ per minutes $)$ and mean minimal systolic arterial pressure were significantly less in Group B $(P=$ $<0.05$ ). In terms of side effects, patients in Group BF had fewer side effects (16.66\%) compared to control group (36.66\%). ${ }^{8}$ Astudy conducted in Iran 2011 assessed the incidence and severity of shivering using intrathecal fentanyl $(25 \mathrm{mcg})$ with $12.5 \mathrm{mg}$ bupivacaine. The total incidence of shivering in Group fentanyl was $10 \%$ compared to $75 \%$ in the control group..$^{20}$ Another study, conducted in Turkey looked at the incidence of hypotension after administration of low-dose bupivacaine with fentanyl $(25 \mathrm{mcg})$ or without fentanyl in elective cesarean section. They found that sensory block was adequate for surgery in all patients. Hypotension occurred in $75 \%$ patients in the fentanyl group and $100 \%$ in the control group. They conclude that the incidence of hypotension, number of ephedrine treatments, and need for ephedrine was significantly greater in the non-fentanyl group. ${ }^{11}$ In contrast, a study by Gajanan et al. found no significant difference in intraoperative hypotension $(94.20 \pm 5.07$ vs $96.90 \pm 6.30$ ), pulse rate $(86.47 \pm 3.30$ vs $87.13 \pm 5.03)$ and respiratory rate $(6.07$ vs 16) between treatment and control group. But $5 \%$ of the treatment group develop mild Pruritis which did not need treatment. ${ }^{21,22}$

Other studies in different parts of Europe and Asia have shown that patients who received intrathecal fentanyl $(25 \mathrm{mcg})$ with Spinal
Bupivacaine had not only improved or prolonged intraoperative analgesic time and reduced analgesic requirements, but also reduced postoperative analgesic requirements for at least $6 \mathrm{hrs} .{ }^{12,16}$ The incidence of nausea and shivering is significantly reduced as well. Postoperative pain relief and hemodynamic stability are increased by adding fentanyl. Pruritis, maternal respiratory depression and changes in Apgar score do not occur, as not affected by low doses of intrathecal fentanyl. ${ }^{21}$ A Nigerian study to compare analgesia efficacy of intrathecal fentanyl for patients undergoing elective open reduction and internal fixation of lower limb fractures (ORIF) found that intrathecal fentanyl provided prolonged analgesia for 4 hours post operatively. In addition, spinal anaesthesia complications were reduced. ${ }^{23}$

Many studies have been performed to compare quality of anaesthesia and postoperative analgesic effect of intrathecal fentanyl and bupivacaine in elective cesarean section. Most of these studies have been conducted in the developed world and in western populations. The presence of racial, cultural, genetic and socio demographic difference in the perception of pain has been well documented, ${ }^{24,25}$ meanwhile intraoperative \& early postoperative pain is a major problem which needs an immediate and sustainable solution.

Mothers who undergo elective cesarean section are more stable in terms of intraoperative complications, general morbidity and mortality than those who undergo emergency cesarean section, ${ }^{26}$ so knowing the effectiveness of intrathecal fentanyl with spinal anesthesia in terms of intraoperative anaesthesia quality and postoperative analgesia helps for better outcomes of the fetus and maternal satisfaction. This research can also help as a back ground for future research on related topic. This study aimed to assess postoperative analgesic effect of intrathecal fentanyl as an adjuvant to spinal anaesthesia in comparison with spinal anaesthesia with bupivacaine only among laboring mothers who undergone emergency cesarean delivery at the University of Gondar Hospital.

\section{Materials and methods}

\section{Study design and period}

Hospital based observational cohort study was conducted from February1 to May 5, 2015.

\section{Study area}

The study was conducted at theUniversity of Gondar Hospital. The University of Gondar hospital is one of the big governmental teaching hospitals in the Amhara region giving services for around five million people. As documents in the hospital statistical office showed, on average 4000 mothers yearly delivered in this hospital. From those $4000,15 \%$ were delivered CS and from this patient population; more than half of them were delivered by SA. After operation diclofenac and tramadol were the drugs used in obstetric ward to manage postoperative pain. Now a days in this hospital, intrathecal fentanyl are used together with bupivacaine to improve intraoperative anaesthesia and quality of postoperative analgesia.

\section{Source population}

All laboring mothers who were delivered by cesarean section at the University of Gondar Teaching hospital.

\section{Study population}

All laboring mothers who were delivered by emergency cesarean section under spinal anaesthesia at the University of Gondar Teaching hospital during the study period. 


\section{Inclusion criteria}

All laboring mothers that undergone emergency cesarean section under spinal anaesthesia and those who had been American society of Anesthesiologist (ASA) class I and II.

\section{Exclusion criteria}

All mothers delivered by cesarean section under spinal anaesthesia with added adjuvant like transversus abdominal plexus (TAP) block other than intrathecal fentanyl, those receiving spinal anaesthesia with lidocaine and those who required systemic analgesia intraoperatively.

\section{Study variables}

Dependent variables: Severity of postoperative pain, first analgesia request time and total analgesia consumption.

\section{Independent variables}

A. Socio-demographic variables: \{age, weight, height\}, Parity, gestational age, duration of surgery, ASA category, time from intrathecal injection to delivery of the child.

B. Factor related to related with spinal anaesthesia: Level of sensory block, onset of sensory block, grade of motor block, onset of motor block, duration of motor block to recover,

C. Factor related with perioperative side effects of spinal anaesthesia: Nausea/vomiting, hypotension, shivering, pruritis, hemodynamic changes, maternal respiratory depression, APGAR score and bradycardia.

\section{Operational definition}

Hypotension: Defined as a Systolic blood pressure of below $90 \mathrm{mmHg}$ or lower than $30 \%$ of starting systolic blood pressure or MAP less than $70 \mathrm{mmHg}{ }^{7}$

Bradycardia: Defined as a heart rate less than 50 beats/minutes. ${ }^{19}$

Respiratory depression: Defined as a respiratory rate less than 10 breaths per minute or oxygen saturation less than $90 \%$ will be consider. $^{7}$

VAS: visual analogue scale which is a method of pain assessmentdetermined by the patient making a mark of their pain intensity on a line which is 100 millimeter long. ${ }^{16}$

\section{No pain worst imaginable pain}

Time for first analgesia request: Defined as the time from intrathecal injection to first request for analgesia. ${ }^{23}$

Total analgesic consumption: Defined as type and amounts of analgesic drugs given to the patient within $12 \mathrm{hrs}$ postoperatively.

Pruritis: Defined as any scratch or itching complained by the patient and its intensity was assessed as mild (itching was only a minor concern), moderate (itching was a primary concern, although bearable, and the patient said she would rather itch than hurt) or severe (unbearable, patient requested treatment).

\section{Sample size calculation and sampling techniques}

All consecutive laboring mothers who delivered by cesarean section under spinal anaesthesia during the study period were included in the study.

\section{Data collection methods}

During each procedure the data collectors observed the intraoperative condition of the patient. Post operatively each patients was interviewed and their chart were also reviewed. Group BF were those laboring mother who had been given $25 \mathrm{mcg}$ of intrathecal fentanyl with $0.5 \%$ of $10 \mathrm{mg}$ bupivacaine. Another group SB those who were given $0.5 \%$ of $12.5 \mathrm{mg}$ of bupivacaine only. One of the trained data collector took and observe intraoperative necessary information who were free from intraoperative anaesthesia monitoring. Starting from the immediate postoperative time, presence and scale of pain, time for the first analgesic request as well as analgesics need were assessed by the other trained data collector. These assessments were done at $1 \mathrm{hr}, 2 \mathrm{hrs}, 3 \mathrm{hrs}$, $4 \mathrm{hrs}$, $5 \mathrm{hrs}$ and $6 \mathrm{hrs}$ for VAS andwithin $12 \mathrm{hrs}$ of postoperative total analgesic consumption was taken. The categories of patients were identified by the by data collector. The VAS was determined by the patient marking their pain intensity on a line which $100 \mathrm{~mm}$ long. Two BSC anaesthetists were selected to collect data and one day training was given on how to collect data. Another MSc anaesthetist was assigned to assist and supervise data collectors.

\section{Data quality control issue}

To ensure the quality of data, pretest of the data collection tool was done on patients by taking 10 respondents $(5 \mathrm{BF}$ group and 5 SB group) who undergone emergency cesarean section under spinal anaesthesia and were not included in the main study. Data was checked for completeness, accuracy and clarity on the day of collection by the principal investigator. Data clean up and cross checking was done before analysis. Training was given for data collectors and supervise for one day on how to approach study subjects and on how to use data collection tool. Supervision by principal investigator and supervising MSc anaesthetist.

\section{Data management and analysis}

Data was checked, coded, entered and analyzed using SPSS version 20 software. Demographic data were analyzed using student test (for normal distribution variables) and Fisher exact test (for categorical variables) as appropriate. The data were tested for normality using Shapiro- Wilk normality test. There was normal distribution data for the time of the first analgesic request and total duration of analgesia as checked using Shapiro-Wilk test and homogeneity of variance assessed using Levene's test for equality of variances. Therefore, an independent t-test was run on the data with $95 \%$ confident interval to analyze measurement of time of the first analgesic request as well as total duration of analgesia time.

However, there was not normally distributed for repeated VAS measurements, and total post-operative analgesic consumption as checked using Shapiro-Wilk test. Therefore, non-parametric Mann Whitney $U$ test was run on the data as well as $95 \%$ confidence interval to analyze with further paired comparison at each time interval. The comparisons of categorical parameters were analyzed using chi-square test or Fisher's exact test. Normally distributed data are presented as mean $\pm \mathrm{SD}$ whereas not normally distributed data presented as median (IQR) and categorical data presented by frequencies (percentages). A $\mathrm{p}$ - value $<0.05$ was considered statistically significant.

\section{Ethical clearance}

Ethical clearance was obtained from Gondar College of Medicine and Health Science, School of Medicine Ethical Review Committee. Written informed consent was obtained from each study subject after clear explanation what they would have to do take part in the research (Figure 1). 


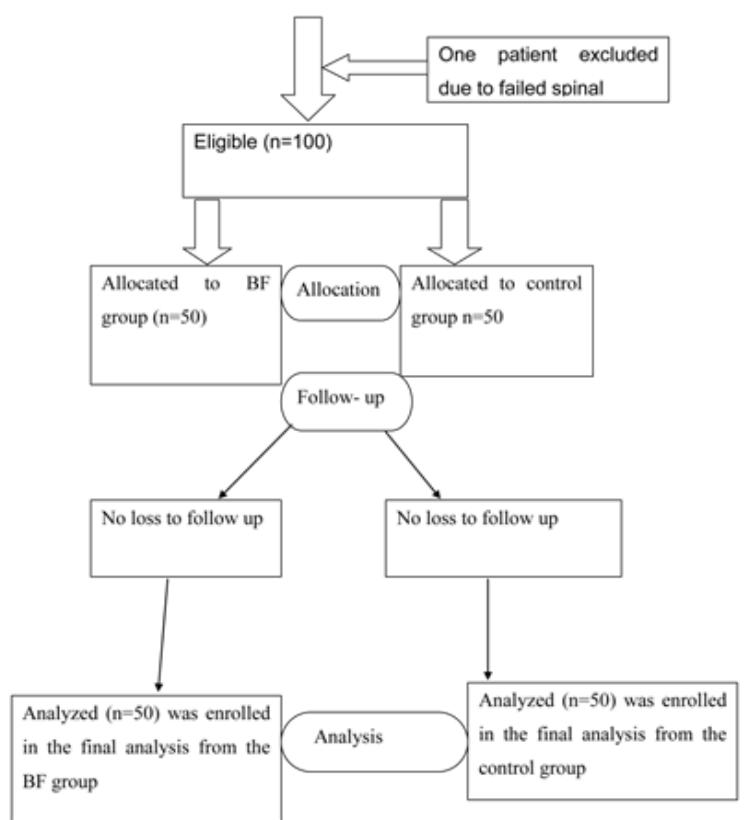

Figure I CONSORT patient flow diagram.

\section{Results}

\section{Socio-demographic characteristics of the participants}

A total of 101 patients were given spinal anaesthesia. One patient was excluded because spinal anaesthesia failed and converted to general anaesthesia. All 100 laboring mothers who underwent caesarean delivery under spinal anaesthesia during the study period were included with a response rate of $99 \%$. Of these patients, 50 mothers were given intrathecal fentanyl $(25 \mathrm{mcg})$ with $(10 \mathrm{mg}) 0.5 \%$ bupivacaine $(\mathrm{BF})$ and 50 patients with $12.5 \mathrm{mg}(0.5 \%)$ bupivacaine (BS group).

The demographic characteristics (age, height, weight, gestational age, duration of surgery) were comparable between the groups as shown in Table 1. Regarding with ASA physical status, $82 \%$ of BF group and $74 \%$ of SB group were ASA I while $18 \%$ of BF group and $26 \%$ of SB group were ASA II. Concerning parity $86 \%$ in BF group and $76 \%$ in BS group were para2 and below, $14 \%$ in BF group and $24 \%$ in BS group were para 3 and above Table 1.

\section{Characteristics of spinal anaesthesia}

There was no difference in highest sensory level, onset of sensory block and onset of motor block. But there was a prolonged motor block in SB group than BF group 272.5 (81) vs.157.5 (59) in minutes. The duration of analgesia (time from subarachnoid injection to first report of pain) was reduced in BF group (275.10 \pm 42.43 minutes) vs. $(156.10 \pm 34.45$ minutes) in BS group (Table 2).

\section{Hemodynamic parameters}

There were no statistical significant in mean heart rate and mean arterial blood pressure at various time interval in both groups as shown in Tables 3 and 4.

\section{Postoperative pain VAS score}

There was no difference on VAS score at $1 \mathrm{hr}$ which was equal to zero between BF group and BS group (Table 5).

Table I Demographic and anaesthetic base line characteristics

\begin{tabular}{llll} 
Group & $\mathbf{B F}(\mathbf{n}=\mathbf{5 0})$ & $\mathbf{B S}(\mathbf{n}=\mathbf{5 0})$ & p- value \\
\hline Age in years & $26(5)^{* *}$ & $27(7)^{* *}$ & 0.844 \\
Weight in kilogram & $60(11.25)^{* *}$ & $60(10.25)^{* *}$ & 0.52 \\
Height in meter & $1.6(0.15)^{* *}$ & $1.58(0.12)^{* *}$ & 0.754 \\
Gestational age in week & $39.06 \pm 1.449^{*}$ & $39.28 \pm 1.457^{*}$ & $0.45 I$ \\
ASA status :- ASA I & $41(82 \%)$ & $37(74 \%)$ & 0.334 \\
ASA 2 & $9(18 \%)$ & $13(26 \%)$ & \\
Parity:- S para 2 & $43(86 \%)$ & $38(76 \%)$ & 0.202 \\
zpara 3 & $7(14 \%)$ & $12(24 \%)$ & \\
duration of surgery in minute & $58.2 \pm 11.185^{*}$ & $55 \pm 10.738^{*}$ & 0.136 \\
Time from intrathecal injection to delivery of child & $15(3)^{* *}$ & $14(3)^{* *}$ & 0.45
\end{tabular}

Category: *: Mean \pm SD, **: Median (IQR).

Table 2 Characteristic of spinal anaesthesia and duration of analgesia

\begin{tabular}{llll}
\hline Group & $\mathbf{B F}(\mathbf{n}=\mathbf{5 0})$ & $\mathbf{B S}(\mathbf{n}=\mathbf{5 0})$ & $\mathbf{p}$ - value \\
\hline Highest sensory level(dermatome) & $\mathrm{T} 4$ & $\mathrm{~T} 4$ & - \\
Onset of sensory block (minute) & $7.14 \pm 1.17$ & $7.22 \pm 1.14$ & 0.732 \\
Grade of onset of motor block(minute) & $5.68 \pm 0.89$ & $5.65 \pm 0.82$ & $0.86 \mathrm{I}$ \\
Duration of grade 0 motor block & $157.5(59)^{*}$ & $272.5(81)^{*}$ & $0.00 \mathrm{I}$ \\
Duration of analgesia(minute) & $275.10 \pm 42.43$ & $156.10 \pm 34.45$ & $0.00 \mathrm{I}$ \\
\hline
\end{tabular}

Values: Mean $\pm \mathrm{SD}$; $*$ : Median (IQR), T4: Dermatome level, p-value $<0.05$ is significant.

Table 3 Mean heart rate at various time intervals

\begin{tabular}{|c|c|c|c|c|c|c|c|c|c|}
\hline \multirow[t]{2}{*}{ Group } & & \multicolumn{8}{|c|}{ Mean Pulse Rate at Various Time Intervals (Beat per minutes) } \\
\hline & time & Baseline & $5 \mathrm{~min}$ & $10 \mathrm{~min}$ & $15 \mathrm{~min}$ & $20 \mathrm{~min}$ & $30 \mathrm{~min}$ & Ihr & $2 \mathrm{hr}$ \\
\hline BF & Mean \pm SD & $99.32 \pm 17.776$ & $93.22 \pm 20.029$ & $84.46 \pm 15.902$ & $86.80 \pm 16.618$ & $87.78 \pm 13.475$ & $87.38 \pm 12.144$ & $86.10 \pm 12.543$ & $86.54 \pm 10.895$ \\
\hline BS & Mean \pm SD & $99.80 \pm 18.748$ & $94.48 \pm 17.59$ & $86.92 \pm 16.249$ & $84.64 \pm 15.243$ & $89.86 \pm 15.653$ & $92.58 \pm|5.77|$ & $91.10 \pm 15.329$ & $94.12 \pm 11.837$ \\
\hline
\end{tabular}

Values are Mean \pm SD, $P$ - value $>0.05$.

Citation: Yesuf KA, Gebremedhn EG, Melkie TB.Analgesic effect of intrathecal fentanyl as an adjuvant to spinal anaesthesia in comparison with spinal anaesthesia with bupivacaine only for mothers delivered by emergency cesarean section.J Anesth Crit Care Open Access. 20I 7;7(5): I-6.

DOI: 10.15406/jaccoa.2017.07.00278 
Table 4 Mean arterial blood pressure at various time intervals

\begin{tabular}{|c|c|c|c|c|c|c|c|c|}
\hline Group & & Mean Arteri & Blood Press & e at Various T & e Intervals ( & $\mathrm{nHg})$ & & \\
\hline & time & $5 \mathrm{~min}$ & $10 \mathrm{~min}$ & $15 \mathrm{~min}$ & $20 \mathrm{~min}$ & $30 \mathrm{~min}$ & Ihr & $2 \mathrm{hr}$ \\
\hline $\mathrm{BF}$ & Mean \pm SD & $77.54 \pm 13.23$ & $73.66 \pm 15.00$ & $72.24 \pm 11.09$ & $72.50 \pm 10.30$ & $69.56 \pm 11.457$ & $72.68 \pm 11.946$ & $76.80 \pm 9.225$ \\
\hline BS & Mean \pm SD & $76.9 \pm 14.710$ & $73.68 \pm 14.63$ & $74.64 \pm 11.008$ & $75.74 \pm 13.03$ & $72.58 \pm 13.04$ & $76.76 \pm 12.603$ & $81.00 \pm 12.202$ \\
\hline
\end{tabular}

Values are Mean $\pm \mathrm{SD}, P$ - value $<0.05$.

Table 5 Postoperative Visual Analogue scale pain scores median (IQR) in millimeters over the first 12 postoperative hours

\begin{tabular}{llll}
\hline Group & $\mathbf{B F}(\mathbf{n}=\mathbf{5 0})$ & $\mathbf{S B}(\mathbf{n}=\mathbf{5 0})$ & $\mathbf{P}-$ value \\
\hline VAS score at $2 \mathrm{hrs}$ & $0(0)$ & $0(20)$ & $0.00 \mathrm{I}$ \\
VAS score at $3 \mathrm{hrs}$ & $0(0)$ & $30(30)$ & $0.00 \mathrm{I}$ \\
VAS score at $4 \mathrm{hrs}$ & $20(30)$ & $30(40)$ & 0.001 \\
VAS score at $5 \mathrm{hrs}$ & $50(30)$ & $20(30)$ & 0.001 \\
VAS score at 6hrs & $10(20)$ & $20(26)$ & 0.023 \\
VAS score at I2 hrs & $20(39)$ & $7.5(30)$ & 0.09 \\
\hline
\end{tabular}

Value in Median (IQR) in millimetres.

\section{Postoperative time for the first analgesic request}

Postoperatively, an independent $\mathrm{t}$ - test showed that the time from intrathecal injection to the first analgesic request was significantly different between the two groups $(P=0.001)$. Mothers in the $\mathrm{BF}$ group had a significant longer time for the first analgesic request compared to the BSgroup, $(301.00 \pm 46.55)$ vs. $(200.20 \pm 44.19)$ minutes as shown in Figure 2.

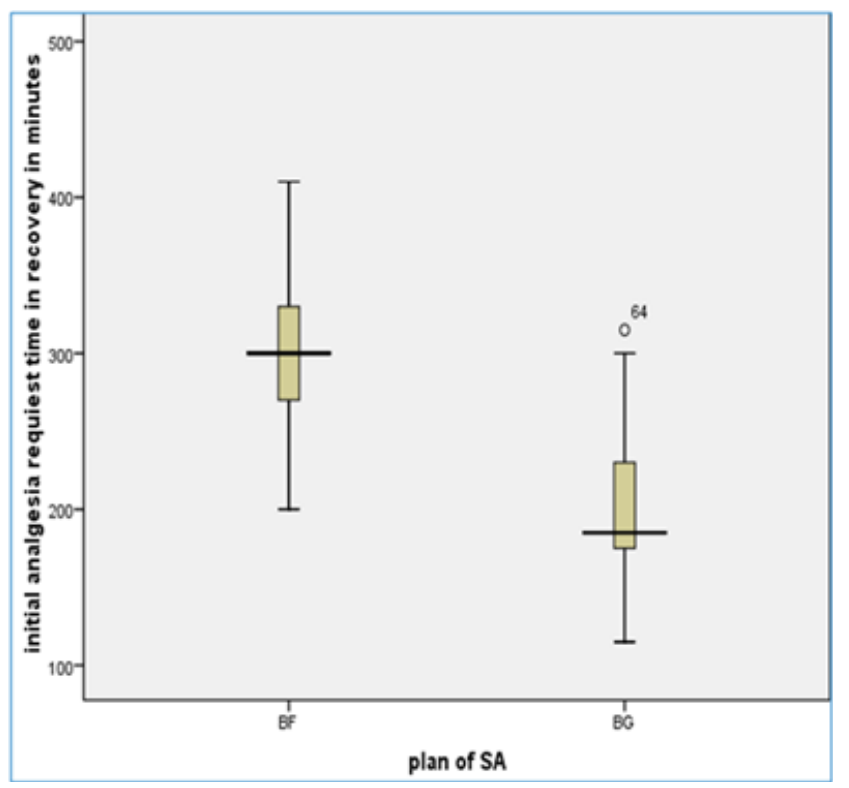

Figure 2 Box and whisker plot graph showed the mean of postoperative analgesia request time.

\section{Postoperative total analgesia consumption}

Since the postoperative $12 \mathrm{hrs}$ of both total diclofenac and total tramadol consumption were not normally distributed as checked by Shapiro-Wilk test, Mann-Whitney $U$ test was used to assess the associations. Patients undergoing intrathecal fentanyl showed a reduced postoperative total tramadol consumption within $12 \mathrm{hrs}(\mathrm{BF}$ vs. BS group), described as median (IQR) $\mathrm{mg}$, at 12 hours $50(50)$ vs. $100(50), P$ value $=0.001$.In the contrary, the total diclofenac consumption increased in BF group than BS within 12hours (BF vs. BS) described as median (IQR) mg, 75(75) vs. 0(75), 0.001 as shown in Figure 3.

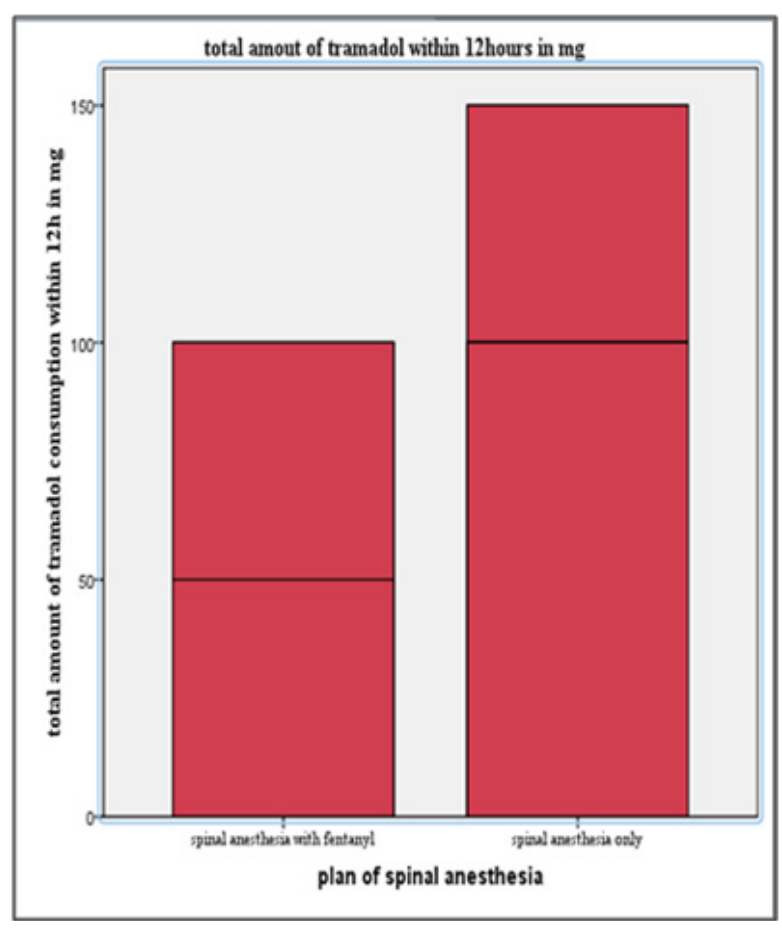

Figure $3 \mathrm{~A}$ bar graph of postoperative total tramadol consumption in each group within $12 \mathrm{hrs}$ of patients.

\section{Incidence of perioperative complications}

No patients developed complications during the early postoperative period. In terms of intraoperative complications, 13(26\%) patients in BF group \& 19(38\%) in BS group were developed hypotension and treated with IV fluid. None of them needed vaso- active drugs. The differences in incidence of hypotension did not reach statistical significance. Three $(6 \%)$ patients in BF \& $18(36 \%)$ in BS group developed nausea, $P=0.001$. Vomiting occurred in one patient in $\mathrm{BF}$ group compared with 12 patients in BS group with p-value $=0.002$. None of group BS patients developed pruritus but $7(14 \%)$ patients in group BF developed mild pruritus, which did not need treatments. Shivering occurred in $15(30 \%)$ patients in BF vs. $29(58 \%)$ in BS group with $\mathrm{p}$ - value of 0.005 . There was no significant difference in total fluid, maternal and neonatal respiratory depressions as well as APGAR score at one and five minutes as shown in Table 6. 
Table 6 Incidence of perioperative complications (category reference:*= mean \pm SD)

\begin{tabular}{llll} 
Complications & BF group(n=50) & BS group(n=50) & p-value \\
\hline Hypotension & $13(26 \%)$ & $19(38 \%)$ & 0.198 \\
Nausea & $3(6 \%)$ & $18(36 \%)$ & $0.00 \mathrm{I}$ \\
Vomiting & $1(2 \%)$ & $12(22 \%)$ & 0.002 \\
Shivering & $15(30 \%)$ & $29(58 \%)$ & 0.005 \\
Pruritis & $7(14 \%)$ & $0(0)$ & 0.006 \\
Respiratory depression (spo2 <90\% or RR <9bpm) & $0 \%$ & $0 \%$ & \\
Fetal bradycardia (HR < I20 bpm) & $0 \%$ & $0 \%$ & 0.414 \\
Total iv fluid given intraop (Its) & $2.00 \pm 1.00 *$ & $2.00 \pm 0.665^{*}$ & 0.748 \\
APGAR score At I minutes & $7-9$ & $7-10$ & 0.939 \\
At 5 minutes & $10-J u l$ & $7-10$ & \\
\end{tabular}

\section{Discussion}

Recent trends in obstetric anaesthesia show an increased usage of intrathecal opioids together with regional anesthesia. ${ }^{5}$ In the context of multimodal analgesia and augmentation strategies for epidural and intrathecal analgesia, the discovery of opioid receptors and subsequent development of the technique of epidural and intrathecal opioid administration is undoubtedly one of the most significant advances in pain management. Studies have shown that spinal opioids can provide profound analgesia with fewer central and systemic adverse effects than the opioids administered systemically and are now used successfully. ${ }^{21,27}$ Administration of fentanyl intrathecally has contributed to improved analgesia during intra-operative as well as the postoperative period in caesarean delivery. Intrathecal fentanyl spreads in the CSF to move into both opioid specific and other nonspecific binding sites in the spinal cord as well as its rostral migration via the cerebrospinal fluid to supra spinal sites. Because of its high affinity with non-specific binding sites on the lipid surface only a small proportion of the administered dose migrates to the cervical region, it does not cause respiratory depression. ${ }^{18,28}$

Many studies demonstrate synergy between opioid's \& local anaesthetic agents. Fentanyl by acting on mu- receptor in spinal cord, it opens $\mathrm{k}+$ channels \& reduced influx of $\mathrm{ca}^{2+}$ so that it inhibits release of neuro transmitters. Opioids act on nociceptive afferent pain pathway, not on sympathetic efferent pathway. Local anaesthetic agents/bupivacaine act by blocking voltage gated $\mathrm{Na}+$ channels in spinal cords. ${ }^{18,19}$

In our study, the addition of $25 \mathrm{mcg}$ fentanyl on $10 \mathrm{mg}$ bupivacaine had no any significant difference on the onset of highest sensory analgesia $(P=>0.05)$. Not only these, but also had no any difference in maximum cephalic spread attained in both groups, which was at T4. A study done by Gauchan et al used $3 \mathrm{ml}$ of $0.5 \%$ bupivacaine with $25 \mathrm{mcg}$ fentanyl and $3 \mathrm{ml} 0.5 \%$ bupivacaine which was a little higher dose compared to our study, they did not found any significant time difference for highest sensory analgesia, which was $(6 \pm 2.5)$ minutes in BF group vs. $(7.9 \pm 2.5)$ minutes in control group. Another study by Gajanan et al using small dose compared to our study ( $20 \mathrm{mcg}$ fentanyl with 10mg bupivacaine) and found that sensory block to reach T4 was achieved after $6.5 \mathrm{~min}$ in those who received fentanyl compared to $8 \mathrm{~min}$ in the control group. In our study, to reach highest sensory analgesia between BF and BS group was $7.14 \pm 1.17$ vs. $7.22 \pm 1.14$ in minutes respectively. The addition of $25 \mathrm{mcg}$ intrathecal fentanyl to $10 \mathrm{mg}$ bupivacaine did not alter the onset of sensory analgesia and height of block which was supported by the above two studies..$^{13,21}$ These might be due to almost similar dose we used in our study. But a study in 2014 by Dhumal PR et al., ${ }^{8}$ to compare synergistic effect of intrathecal fentanyl $25 \mathrm{mcg}$ in addition to $5 \mathrm{mg}$ bupivacaine and
$7.5 \mathrm{mg}$ bupivacaine only, they found that the mean time to reach peak sensory level was earlier with fentanyl group $(5.03 \pm 1.45)$ in minutes than control group $(6.37 \pm 1.33)$ in minutes. ${ }^{8}$ The above study showed a significant difference in mean time of peak sensory analgesia compared to our result $(7.14 \pm 1.17$ in BF group vs. $7.22 \pm 1.14$ in BS group) in minutes, these might be due to we used large dose of bupivacaine in both group as compared to study by Dhumal PR which is the most determinant factor for spread of local anaesthetic agent. ${ }^{29}$

Our study showed that addition of $25 \mathrm{mcg}$ fentanyl increased total duration of complete analgesia (time from intrathecal injection to when the patients complained of any pain, VAS $>0$ ) which was $275.1 \pm 42.4$ in minutes compared to bupivacaine group, $156.10 \pm$ 34.45 in minutes with $\mathrm{p}$ value $=<0.001$. A study done by Shashikala TK et al., ${ }^{22}$ grouped 99 parturients into, FB group who had been given $2 \mathrm{ml}$ of $0.5 \%$ bupivacaine with $12.5 \mathrm{mcg}$ fentanyl and $\mathrm{BC}$ group receiving $2 \mathrm{ml} 0.5 \%$ bupivacaine only. They found that the total duration of mean time analgesia between Group BC and Group FB was $165 \pm 29.8$ minutes in Group BC and 259.4 \pm 35.3 minutes in Group FB respectively. ${ }^{22}$ Relatively total duration of analgesia was longer in our study than the above study, even though patients characteristic and sample size were similar. These might be due to increasing the dose of intrathecal fentanyl will increase the duration of analgesia time. Another study in Nigeria gave $25 \mathrm{mcg}$ intrathecal fentanyl to those patient that undergoing lower limb surgery showed that the time of complete analgesia in Group FB was significantly longer than the SB group with a mean \pm standard deviation of 239.97 \pm 28.58 minutes compared to $129.17 \pm 11.61$ in minutes and $p$-value of $<0.001$ respectively. ${ }^{23}$ Our results are consistent with the above study even though patient characteristics, type of procedure and duration of surgery were differ from our study. These might be due to similar dose of intrathecal fentanyl $(25 \mathrm{mcg})$ we used.

In regard to our study, mothers in the BF group had a significant longer time for the first analgesic request (effective analgesia) compared to mothers in BS group, (301.00 \pm 46.55$)$ vs. (200.20 \pm 44.19) in minutes respectively. Study done by Biswas BN et al used $12.5 \mathrm{mcg}$ intrathecal fentanyl showed that analgesia request time in Group B $(n=20)$ was $150 \pm 10.48$ in minutes and $248 \pm 11.76$ minutes in Group BF $(n=20)$ respectively. ${ }^{18}$ In the above study, analgesia request time was shorter than in our study. This might be due to small sample size and small dose of intrathecal fentanyl $(12.5 \mathrm{mcg})$ they used compared to our study. Other study in Nepal showed time to first analgesia request was short in both groups $134 \pm 5.6$ minute in group BS and $164 \pm 9$ minute in group BF. ${ }^{13}$ Even though they had similar patient characteristics, dose of intrathecal fentanyl and operation. This might be due to experience (perception) of pain is affected by genetics, cultural and social factors in different ethnicity across the world. $^{25}$ 
Onset of motor blockade was comparable in both groups $(5.68$ \pm 0.89 vs. $5.65 \pm 0.82$ ) in minutes and all patients had grade III motor block in our study. Fentanyl has no action on motor blockade 8,18 . There was a prolonged motor block in SB group compared to BF group 272.5 (81) vs. 157.5 (59) minutes with $P$ - value $=0.001$ Study's by Dhumal PR et al. ${ }^{7}$ motor recovery in group BF was 77.2 \pm 14.7 minutes and in Group B was $98.80 \pm 8.91$ minutes respectively which was significant but not similar to our study. This might be due to the small dose of bupivacaine used, (Group B patients with $7.5 \mathrm{mg}$ of $0.5 \%$ heavy Bupivacaine) and in (Group BF patients with $5 \mathrm{mg}$ of $0.5 \%$ heavy Bupivacaine $+25 \mu$ g fentanyl) respectively compared to our study. ${ }^{7}$ Study by Gregory L et al., ${ }^{30}$ to compare $4.5 \mathrm{mg}$ isobaric bupivacaine with $12 \mathrm{mg}$ hyperbaric bupivacaine in addition to either of $50 \mathrm{mcg}$ intrathecal fentanyl of $200 \mathrm{mcg}$ morphine, they found prolonged motor block in hyperbaric bupivacaine group than isobaric bupivacaine (158.6 \pm 44.9 vs. $32 \pm 33.2)$ in minutes respectively.,30 So we conclude that a prolonged motor block in BS group compared to BF group in our study was due to large dose of bupivacaine in BS group.

With regard to our, study there was no difference on VAS score at $1 \mathrm{hr}$ between BF and BS group. This might be due to the analgesic effect of spinal anesthesia that continue in both BS and BF group who had the same value of VAS score at one hour. But there was a reduced median VAS score at ( $2 \mathrm{hr}, 3 \mathrm{hr}, 4 \mathrm{hr}, 5 \mathrm{hr}$ and $6 \mathrm{hours})$ in those patients who had taken $25 \mathrm{mcg}$ intrathecal fentanyl (BF group) with $\mathrm{p}$-value $=<0.05$.The result at $2 \mathrm{hr}, 3 \mathrm{hr}$ and 4 hours was consistent with randomized trial by Cowan CM. et alto compare postoperative analgesia among control group, $\mathrm{BF}$ and bupivacaine dimorphine and they showed that VAS score was similar in the first 3 hours in control group \& BF group but after that their become significant difference. ${ }^{16}$ This similarity might be due all most the same dose of intrathecal fentanyl used for similar procedure.

The median VAS scores at $5 \mathrm{hrs}$ and $6 \mathrm{hrs}$ was significantly reduced in BF group than BS group with $\mathrm{p}$ - value $<0.05$. Our finding was comparable with a randomized study conducted in Nigeria to compare the analgesic effect of $25 \mathrm{mcg}$ intrathecal fentanyl with bupivacaine only for lower limb surgery. ${ }^{23}$ This similarity might be related with the wear off analgesic effects of spinal anesthesia in bupivacaine group and the synergistic effect of intrathecal fentanyl with bupivacaine in BF group continued since the duration of intrathecal fentanyl is expected to stay 6hours. After 6hour there was no significant difference in median VAS scores since both groups were treated with diclofenac and tramadol as well as wear of action of intrathecal fentanyl.

Patients in BF had a reduced postoperative total tramadol consumption within $12 \mathrm{hrs}$ compared to BS group, $P=0.001$. Our finding was comparable with a randomized study conducted by Manullang et al showed that intrathecal fentanyl decreased morphine requirements in the first $6 \mathrm{~h}$ with $P$ - value $=0.01 .{ }^{31}$ The reason might be patient in $\mathrm{BF}$ group had prolonged duration of analgesia (effective analgesia) than patient in BS group.

Intraoperative nausea and vomiting occurs in more than $66 \%$ of Caesarean deliveries. This is mainly related to peritoneal traction and exteriorization of the uterus performed with regional anaesthesia..$^{21,31}$ In addition, sympathetic cardio accelerator fibers block with high dose of plain bupivacaine, can result in hypotension. This is strongly related to nausea and vomiting. ${ }^{18}$ In regard to our study, $3(6 \%)$ patients in BF group \& 18(36\%) patients in BS group developed nausea and only one patient in BF group \& 12 patients in BS group had vomiting, statistically significant.A study from Iran found that adding $25 \mathrm{mcg}$ fentanyl reduced the incidence of nausea ( 7 patients) and vomiting (4 patients) in BF group compared with 15 patient developed nausea and 12 patients developed vomiting in their BS group. Our study is comparable with these results and our possible explanation for the reduction of nausea/vomiting in BF group is due to increased dose of fentanyl $(25 \mathrm{mcg})$, better quality of analgesia and good hemodynamic stability in BF group. ${ }^{32}$

In our study there were no statistical significant in mean heart rate and mean arterial blood pressure at various time intervals in both groups, which was similar finding with Dhumal PR, et al. and Shashikala TK et al., ${ }^{22}$ However, a study in Nepal showed that the incidence of bradycardia was $5.7 \%$ in control Group and $2.8 \%$ in Group BF with no significant variation in the group..$^{13} \mathrm{~A}$ similar study in Texas showed that bradycardia occurred 3 patients in treatment group and 4 patients in control group which was not significant. ${ }^{19}$ In comparison to the above two studies there were no cases of bradycardia in our patients. This might be due to case selection difference (our cases were emergency cesarean section compared to the above studies which were elective cases), so that there was a continuous labour pain which may have caused sympathetic nervous system stimulation in our case.

In terms of intra-operative hypotension, our study showed that $13(26 \%)$ patients in BF group \& 19(38\%) in BS group developed hypotension which was treated with IV fluid. None of them needed vaso- active drugs. Our finding was similar to that of Olanrewaju NA, et al. ${ }^{23}$. In his study eight of the patients in his control group BS $(26.67 \%)$ group vs. six patients (20\%) in FB group had hypotension that required rapid infusion of crystalloid. ${ }^{23}$ Another controversial study by Harbhej $\mathrm{S}$ et al., ${ }^{19}$ showed $43 \%$ of patients in their treatment group developed hypotension compared with $14 \%$ of patients in control group. ${ }^{19}$ More patients developed hypotension in their fentanyl group than in our study. Their explanations for increased incidence of hypotension was due to higher sensory level was achieved in fentanyl group than BS group. But intrathecal opioids added on local anaesthetic drugs does not potentiate bupivacaine on efferent sympathetic pathway. Even if our study is not statistically significant it is clinically important in our setup since we do not have vaso active drugslike ephedrine and phenylephrine to treat hypotension. Intrathecal fentanyl could be the only option to prevent hypotension. ${ }^{23}$

The effect of intrathecal fentanyl on shivering is because it is highly ionized, lipophilic $\mu$ - receptor agonist and unionized component is rapidly transferred into the spinal cord to act the thermo-regulator and affect spinal afferent thermal inputs at the spinal cord. In our study, shivering occurred in $15(30 \%)$ patients in BF vs. $29(58 \%)$ in BS group with $P$-value of 0.005 . A study by Ali Sadegh et al showed 22 patients (55\%) in Group BS had shivering during recovery and no patient in Group BF had shivering. ${ }^{20}$ In our study, more patients in BF group developed shivering compared to study by Ali. This might be due to different factor like environmental factor and set up of the operation room.

Pruritus induced by intrathecal fentanyl is not due to histamine release but is likely due to the cephalic migration of the opioid in CSF and its subsequent interaction with opioid receptors in the trigeminal nucleus. In our study, none of group BS patients developed pruritus but $7(14 \%)$ in group BF developed mild pruritus, which did not need treatment. A similar study showed thatmild itching was observed in $2(5 \%)$ patients receiving intrathecal fentanyl without any rash and it subsided without any treatment. ${ }^{21}$ Another study in India using $12.5 \mathrm{mcg}$ fentanyl with $10 \mathrm{mg}$ bupivacaine compared with $10 \mathrm{mg}$ bupivacaine, they found that no patients had pruritis in either of the group.$^{18}$ Our study showed more pruritis compared to the above studies. This might be due to an increased dose of fentanyl, $25 \mathrm{mcg}$ compared to the dose of $12.5 \mathrm{mcg}$. 
APGAR scores were similar between two groups in our study at 1minute which was $7-9$ in BF group vs. 7- 10 in BS group and at 5 minutes $7-10$ in both groups respectively. Even though APGAR scores is not a highly sensitive means of neonatal assessment. Umbilical cord blood gas analysis and neonatal neuro-behavioral scores may have revealed more subtle effects. ${ }^{31}$ A study in India using small dose of $(12.5 \mathrm{mcg})$ fentanyl compared to our study $(25 \mathrm{mcg})$ showed that, there were no significant APGAR score difference at 1 and 5minutes which was, 8 -9, 9-10 in control group and 7-9, 9-10 in BF group respectively. ${ }^{18} \mathrm{~A}$ similar study by Gajanan et al., ${ }^{21}$ using $25 \mathrm{mg}$ intrathecal fentanyl in elective cesarean section also showed no APGAR score differences. ${ }^{21}$ Even if our cases were emergency cesarean section compared to the above two studies, our study did not show significant Apgar scores between the groups. Also there was no neonatal bradycardia or respiratory depression noticed in our study. These showed that use of $25 \mathrm{mcg}$ intrathecal fentanyl seems safe in emergency cesarean section.

In our study, none of the patients developed respiratory depression $\left(\mathrm{spo}_{2}<90 \%\right)$ which was supported by many studies. ${ }^{13,18,22}$ This might be due to high affinity of fentanyl with nonspecific binding sites on the lipid surface only a small proportion of the administered dose migrates to the cervical region..$^{33}$

\section{Limitation of the study}

Anaesthesia management may differ from anaesthetist to anaesthetist depending on seniority.It was difficult to properly blind data collection. During data reviewing, it was possible to tell which subjects were in which group so this could be a source of possible bias. It is difficult to evaluate severity of pain for $12 \mathrm{hrs}$ for most subjects, VAS score was also difficult to understand well because of most of our patients are illiterate. Not only this but also it is difficult to get information about time from diagnosis for $\mathrm{C} / \mathrm{S}$ to operation.

\section{Strength of the study}

Subjects were homogeneous (obstetric and emergency patients) which should provide representative results. There was adequate sample size with a planned, estimated and fixed period of time.

\section{Conclusion}

The result of our study demonstrates that the use of intrathecal fentanyl with bupivacaine for cesarean section would improve the quality of anaesthesia, good hemodynamic stability, intraoperative and post-operative analgesia. Not only these but also it reduces analgesia requested time, severity of pain as well as total analgesia consumption without an effect on the mother and fetus. We recommend the use of intrathecal fentanyl with plain bupivacaine, to improve intraoperative quality of anesthesia and early postoperative analgesia for emergency cesarean section in obstetric ward with feedback from care providers using checklist about the possible postoperative fetal effect of fentanyl. We also recommended further randomized control trial in order to avoid bias and to assess the exact severity of pain.

\section{Acknowledgements}

We thank the university of Gondar financial support.

\section{Conflicts of interest}

Author declares there are no conflicts of interest.

\section{Funding}

None.

\section{References}

1. Najmeh M, Elham H, Fariba $M$, et al. The preference of Iranian women to have normal vaginal or cesarean deliveries. JRMS. 2013;18(11):943-950

2. Bruce MG, Rahel G, Alexander G. Low-dose bupivacaine-fentanyl spinal anesthesia for cesarean delivery. RAPM. 2000;25(3):235-239.

3. Fadıl HMO, Yılmaz Y, Meltem K, et al. Spinal anesthesia for elective cesarean section is associated with shorter hospital stay compared to general anesthesia. CTs. 2013;25(2):55-63.

4. Mai EN, Mohammed AM, Ahmed GMA. A comparative study of general anesthesia versus combined spinal-epidural anesthesia on the fetus in cesarean section. EJA . 2013;30:155-160.

5. Jaishri B, Nap S. Synergistic effect of intrathecal fentanyl and bupivacaine in spinal anesthesia for cesarean section. BMC Anesthesiology. $2005 ; 5(1): 5$.

6. Farhat NKS. Complications of Spinal Anaesthesia in Caesarean Section.

7. Dhumal PR, Gunjal VB, Kurhekar VA. Synergistic effect of intrathecal fentanyl and bupivacaine in spinal anesthesia for cesarean section. $B M C$ Anesthesiology. 2005;5(5).

8. Dhumal PR, Gunjal VB, Kurhekar VA. Synergistic effect of intrathecal fentanyl and bupivacaine combination for cesarean section. IJPBR. 2013;4 (1):50-56.

9. Weigl W, Bierylo A, Krzemien Wiczynska S, et al. Comparative study of postoperative analgesia after intrathecal administration of bupivacaine with fentanyl or morphine for elective Caesarean section. Anestezjol Intens Ter. 2009;41(1):28-32.

10. Anjali KN, Prasad GPC. Comparative study of intrathecal hyperbaric Bupivacaine with Clonidine, Fentanyl and Midazolam for quality of anaesthesia and duration of post operative pain relief in patients undergoing elective caesarean section. PJSR. 2011;5(1).

11. Turhanoglu S, Kaya S, Erdogan H. Is there an advantage in using low-dose intrathecal bupivacaine for cesarean section. J Anesth. 2009;23(3):353-357.

12. Rauch E. Intrathecal Hydromorphone for Postoperative Analgesia After Cesarean Delivery, A Retrospective Study. AANA Journal. 2012;80(4):S25-S32.

13. Gauchan S, Thapa C, Prasai A, et al. Effects of intrathecal fentanyl as an adjunct to hyperbaric bupivacaine in spinal anesthesia for elective caesarean section. Nepal Med Coll J. 2014;16(1):5-8.

14. Khezri MB, Rezaei M, Delkhosh Reihany M,et al. Comparison of Postoperative Analgesic Effect of Inrathecal Clonidine and Fentanyl Added to Bupivacaine in Patients Undergoing Cesarean Section: A Prospective Randomized Double-Blind Study. Pain Res Treat. 2014;1-7.

15. Khezri MB, Yaghobi S, Hajikhani M, Asefzadeh S (2012) Comparison of postoperative analgesic effect of intrathecal magnesium and fentanyl added to bupivacaine in patients undergoing lower limb orthopedic surgery. Acta Anaesthesiologica Taiwanica 50(1): 19-24.

16. Cowan CM1, Kendall JB, Barclay PM, et al. Comparison of intrathecal fentanyl and diamorphine in addition to bupivacaine for Caesarean section under spinal anaesthesia. BJA. 2002;89(3):452-458.

17. Choi DH, Ahn HJ, Kim MH. Bupivacaine-sparing effect of fentanyl in spinal anesthesia for cesarean delivery. RAPM . 2000;25(3):240-245.

18. Biswas BN, Rudra A, Bose BK, et al. Intrathecal fentanyl with hyperbaric bupivacaine improves analgesia during caesarean delivery and in early post-operative period. IJA. 2002;46(6):469-472.

19. Singh H1, Yang J, Thornton K, et al. Intrathecal fentanyl prolongs sensory bupivacaine spinal block. Can J Anaesth. 1995;42(11):987-991. 
20. Ali S NF, Bita E. Intrathecal fentanyl for prevention of shivering in spinal anesthesia in cesarean section. MJIRI . 2012;26(2):85-89.

21. Chavan G, Chavan A, Ghosh A. Effect of Intrathecal Fentanyl on subarachnoid block with $0.5 \%$ hyperbaric bupivacaine. International $J$ of Healthcare and Biomedical Research . 2014;2(4):67-76.

22. Shashikala TK, Srinivas VY. A clinical study of effect of spinal fentanyl on subarchnoid block in parturients. JEMDS. 2014;3(34):8980-8990.

23. Olanrewaju NA, Patience TS, Adesuwa SL, et al. Analgesic Effects of Intrathecally Administered Fentanyl in Spinal Anaesthesia for Lower Limb Surgery. MJMS. 2013;6(3):255-2560.

24. Martinez KA, Snyder CF, Malin JL, et al. Is Race/Ethnicity Related to Presence or Severity of Pain in Colorectal and Lung Cancer. J Pain Symptom Manage. 2014;48(6):1050-1059.

25. Post LF, Blustein J, Dubler NN. Pain: Ethics, Culture, and Informed Consent to Relief. J Law Med Ethics. 1996;24(4):348-359.

26. Asifa GFK, Ayesha MH, Tehmina A, et al. maternal morbidity in emergency versus elective caesarean section at tertiary care hospital. $J$ Ayub Med Coll Abbottabad. 2012; 24(1):10-13.

27. Sawi W, Choy YC. A comparative study of postoperative analgesia, side effects profile and patient satisfaction using intrathecal fentanyl with and without morphine $0.1 \mathrm{mg}$ in caesarean section. Middle East $J$ Anaesthesiol. 2013;22 (1):21-26.
28. Karaca F, Erkilic E, Akdikan A, et al. Assessment of the Effect of Intrathecal Low Dose Levobupivacaine or Bupivacaine Combined with Fentanyl in Patients Undergoing Cesarean Section. J Anesth Clin Res. 2014; 5(11).

29. Hocking G, Wildsmith JA. Intrathecal drug spread : Review article. BJA. 2004;93(4):568-578.

30. Gregory L, Bryson FR, Robert M, et al. Small dose spinal bupivacaine for Cesarean delivery does not reduce hypotension but accelerates motor recovery. Can J Anaesth. 2007;54(7):531-537.

31. Manullang, Theodore R, Viscomi, et al. Intrathecal Fentanyl Is Superior to Intravenous Ondansetron for the Prevention of Perioperative Nausea During Cesarean Delivery with Spinal Anesthesia. Anesthesia \& Analgesia. 2000;90(5):1162-1166.

32. AliNasrin FTK, Bita E. Intrathecal fentanyl for prevention of shivering in spinal anesthesia in cesarean section. MJIRI. 2012;26(2):85-89.

33. Arai YCP, Ogata J, Fukunaga K, et al. The effect of intrathecal fentanyl added to hyperbaric bupivacaine on maternal respiratory function during Cesarean section. Acta Anaesthesiol Scand. 2006;50(3):364-367.

34. Filiz K EE, Alev A, Tülin G, et al. Assessment of the Effect of Intrathecal Low Dose Levobupivacaine or Bupivacaine Combined with Fentanyl in Patients Undergoing Cesarean Section. J Anesth Clin Res . 2014;5(11). 